\title{
MULTI SPLIT FUNCTIONAL MODEL OF GEODETIC OBSERVATIONS IN DEFORMATION ANALYSES OF THE OLSZTYN CASTLE
}

\author{
Marek Hubert ZIENKIEWICZ ${ }^{1) *}$, Karolina HEJBUDZKA ${ }^{2)}$ and Andrzej DUMALSKI ${ }^{3)}$ \\ 1) Department of Geodesy and Oceanography, Gdynia Maritime University, Sędzickiego 19, 81-374 Gdynia, Poland \\ 2) STUDIO A+G architecture, photography, scanning 3D, geodesy, 10-719 Olsztyn, Poland \\ 1) Institute of Geodesy, University of Warmia and Mazury, Oczapowskiego 1, 10-957 Olsztyn, Poland \\ *Corresponding author's e-mail:m.zienkiewicz@wn.am.gdynia.pl
}

\section{ARTICleinfo}

Articlehistory:

Received 27 September 2016

Accepted 27 January 2017

Available online 9 February 2017

Keywords:

$\mathrm{M}_{\text {split(q) }}$ estimation

Split functional model

Deformation analyses

Leveling network

\begin{abstract}
The paper presents monitoring of the geodetic displacements using the $M_{\text {split(q) }}$ estimation method. Generally, the approach is based on a multi split functional model of geodetic observations. A typical property of $\mathrm{M}_{\text {split(q) }}$ estimation is that the estimates of the controlled point coordinates are determined by using one observation set in all measurement campaigns. In this paper the authors point out that this method may be particularly useful for adjustment of the surveying network with a low level of the mutual control observations. The precise geometric leveling measurements were used as a dataset for verification of the proposed method efficacy. As a test object the Olsztyn Castle in Poland was taken. The results of the study were compared to the classical method of the least squares estimation. Experiment results showed the important advantages of method of the estimation parameters in a split functional model of geodetic observations.
\end{abstract}

\section{INTRODUCTION}

One of the most important topics in geodesy is identification of observed points' position changes using geodetic networks adjustment, known, in the geodetic terminology as displacement. The methodology of the process requires registration of the controlled point position in at least two measurement epochs (e.g. Caspary, 1988; Duchnowski, 2010; Kamiński and Nowel, 2013; Nowel and Kamiński, 2013). Determining of the deformation indicators, such as displacements of the controlled points, is a complex process, requiring proper surveying equipment, field measurement methods and the optimal processing approach. The selection of the suitable method mainly depends on the type of observations, its accuracy, the size and type of the control geodetic network. The control networks can be divided into two main groups. In the first one, some control points are located outside of the deformation area. Those points can be considered as a stable and treated as references. In the second network type, all of the points may be displaced. In the geodetic terminology, those two groups are known as absolute networks and the relative networks, respectively (Baselga et al., 2015; Amiri-Simkooei, 2016). In the case of the absolute control networks verification of the reference points stability should precede measured points displacement estimation (Aydin, 2012; Cymerman et. al., 2016; Hekimoglu et. al., 2010; Śtroner et al., 2014; Velsink, 2015). The reliability of the geodetic deformation analysis largely depends on the stability of the reference datum (see eg., Sušić et al., 2015; Duchnowski, 2011; Nowel, $2015 \mathrm{a}, 2015 \mathrm{~b}$ ) and is closely linked to the theory of geodetic networks' reliability.

That concern has, over the past decade, made this area the main topic of multiple detailed research studies. The most popular algorithms for the reference points' stability are: robust M-estimation principles (Nowel and Kamiński, 2014; Nowel 2015a, 2015b), hybrid M-estimation (Czaplewski and Wiśniewski, 2008; Zienkiewicz and Bałuta, 2013), classical least squares method (Chen, 1983; Erdogan and Hekimoglu, 2014) and rank tests based ones (Duchnowski 2010, 2011, 2013). On the other hand, the satisfactory results of the geodetic deformation analysis can be obtained, despite the instability of the reference datum, by applying a virtual functional model in $\mathrm{M}_{\text {split }}$ estimation (Zienkiewicz, 2014; Zienkiewicz and Baryła, 2015; Wiśniewski and Zienkiewicz, 2016). This concept is based on assumption that outliers, which are generated by 


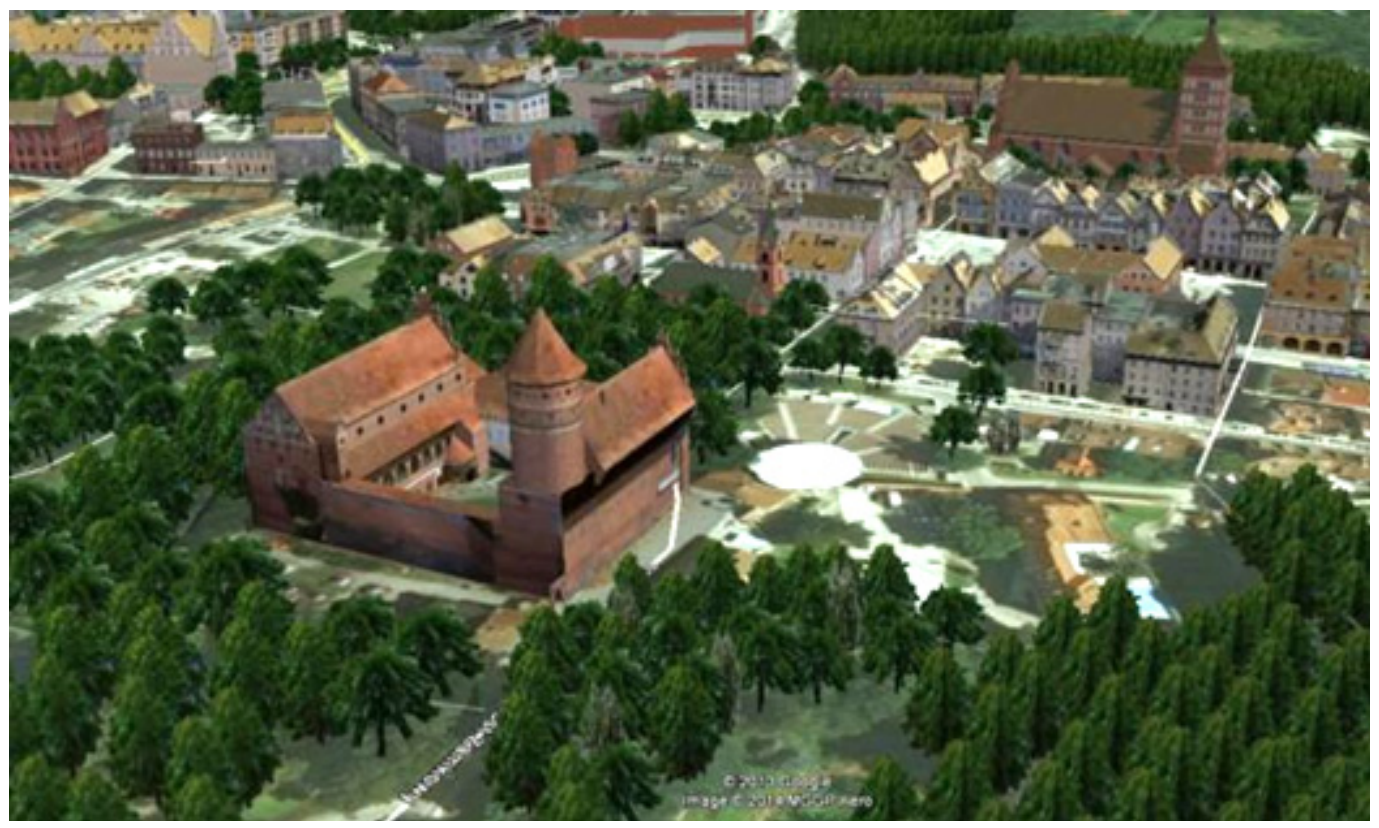

Fig. 1 The tested object - The Olsztyn Castle.

unstable reference points, can be assigned to an additional functional model.

Geodetic network reliability largely depends on the number and location of redundant observations in a network (Prószyński, 1994, 1997; Yetkin and Barber, 2013). Additional observations provide control and improve model accuracy. The reliability is determined by the network geometry. The papers (Hekimoglu et. al., 2011; Hekimoglu and Erdogan, 2012) noted that during the design of measurement network the approach based on a median to detect the points, which are the weakest and the strongest elements of the network configuration, can be used. Considering few variants of the quantity of displaced control points and creating a combination of subnetworks, it is possible to "strengthen" the weakest points of the monitored network by adding additional observations. This approach enables optimal designing of the geodetic network, however, designing both high internal and external reliability is not always possible. The unfavourable position of the monitored object is the main culprit here. This paper demonstrates such network, designed to determine the deformation of the Olsztyn Castle (Fig. 1).

The increase in the redundant observations should improve the reliability of the observations. Lets consider an observation vector, containing the measurement results of two or several measurement epochs, $\mathbf{y}=\mathbf{y}_{1} \cup \mathbf{y}_{2} \cup \ldots \cup \mathbf{y}_{q}$ (where $\mathbf{y} \in \mathfrak{R}^{n}$ indicates the observation vector, and $q$ indicates measurement period). This observation vector, combining measurement from $q$ epochs, is de facto a vector of $q$ competitive random variables $Y_{1} \sim P_{X_{1}}$, $Y_{2} \sim P_{X_{2}}, \ldots, Y_{q} \sim P_{X_{q}}$. The variables may differ with at least the expected value $E\left(Y_{1}\right), E\left(Y_{2}\right), \ldots, E\left(Y_{q}\right)$. Thus, in order to determine the coordinates of controlled points at each epoch, one can use the method of estimation parameters in a split functional model ( $\mathrm{M}_{\text {split(q) }}$ estimation).

This paper is a continuation of the studies included in the publications (Zienkiewicz, 2014; Zienkiewicz and Baryła, 2015; Wiśniewski and Zienkiewicz, 2016). The main aim of this paper is to present the application properties of the $M_{\text {split(q) }}$ estimation in deformation analysis based on geodetic levelling network. An example included in this paper suggests another potential application of the $\mathrm{M}_{\text {split(q) }}$ estimation to engineering issues. Previous studies demonstrated the use of a split functional model to create the point clouds from terrestrial and airborne laser scanning (Błaszczak-Bąk et. al., 2015; Janowski and Rapiński, 2013), coordinate transformation (Janicka and Rapiński, 2013), direct determination of shifts between parameters (Duchnowski and Wiśniewski, 2012, 2014; Wiśniewski and Zienkiewicz, 2016), and determination the deformations indicators of the geodetic networks with unstable reference datum (Zienkiewicz, 2014, 2015; Filipiak-Kowszyk and Kamiński, 2016; Wiśniewski and Zienkiewicz, 2016). This method was also considered as a method of robust estimation (Wiśniewski, 2009a; Ge et. al., 2013). In this paper we propose the application of the $\mathrm{M}_{\text {split(q) }}$ estimation to monitoring the condition of the object, using any number of measurement epochs. This method based on the splitting of the conventional functional model can be considered as a supplement to the classical strategy of the displacement monitoring of the controlled points in the geodetic networks. As a tested object the Olsztyn Castle was chosen. Near its area the absolute leveling network was stabilized and measured by the precise geometric levelling to determine deformation indicators of the Olsztyn Castle. The results of five measurement campaigns 
were taken to the analysis. The conducted empirical analyzes were extended by variants involving the displacement of one and two controlled points. The obtained $\mathrm{M}_{\text {split(q) }}$ estimate results were compared to the results from the least squares adjustment method (LS).

\section{THE THEORETICAL BACKGROUND OF THE SQUARED M SPLIT(Q) $_{\text {ESTIMATION }}$}

In the classical estimation the following traditional functional model of geodetic observation is used

$\mathbf{v}=\mathbf{A X}-\mathbf{y}$

where $\mathbf{A} \in \mathfrak{R}^{n, r} \quad$ - is the known coefficient matrix, $\mathbf{X} \in \mathfrak{R}^{r}$ - denotes unknown parameter vector, $\mathbf{v} \in \mathfrak{R}^{n}$ - is the theoretical corrections of the observation vector. In the $\mathrm{M}_{\text {split(q) }}$ estimation method we assign observation to one of several functional models (Wiśniewski, 2008, 2009a, 2009b, 2009c, 2010):

$$
\mathbf{v}=\mathbf{A X}-\mathbf{y} \stackrel{\text { split }}{\longrightarrow}\left\{\begin{array}{c}
\mathbf{v}_{(1)}=\mathbf{A} \mathbf{X}_{(1)}-\mathbf{y} \\
\vdots \\
\mathbf{v}_{(q)}=\mathbf{A} \mathbf{X}_{(q)}-\mathbf{y}
\end{array}\right.
$$

where $\mathbf{X}_{(1)}, \ldots, \mathbf{X}_{(q)}$ are the competitive versions of the parameter $\mathbf{X}$, whereas $\mathbf{v}_{(1)}, \ldots, \mathbf{v}_{(q)}$ are competing versions of the vector of the theoretical corrections for the same observation vector $\mathbf{y}$. In the deformation analysis of a geodetic network, competitive functional models address one of the $q$ measurement epochs. In the estimation process, each observation $y_{i}$ will be "intrinsically" assigned to the appropriate functional model. Therefore, in contrast to R - estimation and methods related to the principles of classical M estimation, there is no need to organise the collections implementation of specific random variables.

A characteristic property of the $\mathrm{M}_{\text {split(q) }}$ estimation is that the competitive version of the parameters $\mathbf{X}$ are determined using vector $\mathbf{y}$. This vector contains realizations of a several random variables (different observation epochs) with various probability distribution. In the presented method the observations which are related to the particular measuring epoch (campaign) are assigned to the suitable functional model (2). $\mathrm{M}_{\text {split(q) }}$ estimation is follows the assumption that each observation $y_{i}$ can be assigned a $q$ certain amount $K$ indicating the possibility of identifying this observation with one of the random variables. This value is called the elementary split potential (Wiśniewski, 2009a, 2010). For the random variables with the density functions $f\left(y_{i} ; \mathbf{X}\right)$, the split potential for a single observation, at the parameters $\mathbf{X}-\stackrel{\text { split }}{\longrightarrow}\left(\mathbf{X}_{(1)}, . ., \mathbf{X}_{(q)}\right)$, is defined as (Wiśniewski, 2010):

$$
\begin{aligned}
K\left(y_{i} ; \mathbf{X}_{(1)} \ldots, \mathbf{X}_{(q)}\right) & =f\left(y_{i} ; \mathbf{X}_{(1)}\right)^{I_{f}\left(y_{i} ; \mathbf{X}_{(2)}, \ldots, \mathbf{X}_{(q)}\right)}= \\
& =f\left(y_{i} ; \mathbf{X}_{(2)}\right)^{I_{f}\left(y_{i} ; \mathbf{X}_{(1)}, \mathbf{X}_{(3)}, \ldots, \mathbf{X}_{(q)}\right)}=(3) \\
& =\ldots=f\left(y_{i} ; \mathbf{X}_{(q)}\right)^{I_{f}\left(y_{i} ; \mathbf{X}_{(1)}, \ldots, \mathbf{X}_{(q-1)}\right)}
\end{aligned}
$$

where

$$
\begin{gathered}
I_{f}\left(y_{i} ; \mathbf{X}_{(1)}, \ldots, \mathbf{X}_{(j-1)}, \mathbf{X}_{(j+1)}, \ldots, \mathbf{X}_{(q)}\right)=\prod_{l=1, l \neq j}^{q} I_{f}\left(y_{i} ; \mathbf{X}_{(l)}\right)= \\
=\prod_{l=1, l \neq j}^{q}\left[-\ln f\left(y_{i} ; \mathbf{X}_{(l)}\right)\right]
\end{gathered}
$$

is the total $f$-information which is provided by the observation $y_{i}$ after replacing the density function $f\left(y_{i} ; \mathbf{X}_{(j)}\right)$ by all other competitive functions $f\left(y_{i} ; \mathbf{X}_{(l)}\right)$, at $l=1,2, \ldots, q$ and $l \neq j$ (Wiśniewski, 2010). Obtaining the split potential in the whole observation vector $\mathbf{y}$ (global split potential), is possible by defining the product of the elementary potentials of all observations (Wiśniewski, 2010):

$K\left(\mathbf{y} ; \mathbf{X}_{(1)}, \ldots, \mathbf{X}_{(q)}\right)=\prod_{i=1}^{n} K\left(y_{i} ; \mathbf{X}_{(1)}, \ldots, \mathbf{X}_{(q)}\right)$

The optimization problem of the $\mathrm{M}_{\text {split(q) }}$ estimation is formulated on the basis of the global split potential. Namely, for each $\mathrm{M}_{\text {split(q) }}$ estimates of parameters $\mathbf{X}_{(1)}, \ldots, \mathbf{X}_{(q)}$ there are such values $\hat{\mathbf{X}}_{(1)}, \ldots, \hat{\mathbf{X}}_{(q)}$, for which the split potential of the whole set of observation takes the greatest value as follow:

$\max _{\mathbf{x}_{(1)}, \ldots, \mathbf{X}_{(q)}} K\left(\mathbf{y} ; \mathbf{X}_{(1)}, \ldots, \mathbf{X}_{(q)}\right)=K\left(\mathbf{y} ; \hat{\mathbf{X}}_{(1)}, \ldots, \hat{\mathbf{X}}_{(q)}\right)$

The optimization criterion can be replaced with its equivalent form:

$\max _{\mathbf{x}_{(1)}, \ldots, \mathbf{X}_{(q)}} K_{\ln }\left(\mathbf{y} ; \mathbf{X}_{(1)}, \ldots, \mathbf{X}_{(q)}\right)=K_{\ln }\left(\mathbf{y} ; \hat{\mathbf{X}}_{(1)}, \ldots, \hat{\mathbf{X}}_{(q)}\right)$

where

$$
\begin{aligned}
K_{\ln }\left(\mathbf{y} ; \mathbf{X}_{(1)}, \ldots, \mathbf{X}_{(q)}\right) & =\ln K\left(\mathbf{y} ; \mathbf{X}_{(1)}, \ldots, \mathbf{X}_{(q)}\right)= \\
& =\sum_{i=1}^{n} \ln K\left(y_{i} ; \mathbf{X}_{(1)}, \ldots, \mathbf{X}_{(q)}\right)
\end{aligned}
$$

A logarithmic function built with the use of the split potential can be transformed to the informative function using the expression (4). Thus, the optimization criterion of the $\mathrm{M}_{\text {split(q) }}$ estimation can be written in the following form (Wiśniewski, 2010):

$$
\begin{aligned}
& K_{\ln }\left(\mathbf{y} ; \mathbf{X}_{(1)}, \ldots, \mathbf{X}_{(q)}\right)=\max \Leftrightarrow \\
& I_{f}\left(\mathbf{y} ; \mathbf{X}_{(1)}, \ldots, \mathbf{X}_{(q)}\right)=\sum_{i=1}^{n} I_{f}\left(y_{i} ; \mathbf{X}_{(1)}, \ldots, \mathbf{X}_{(q)}\right)=\min
\end{aligned}
$$

or in the more general form, replacing $-\ln f(\mathbf{y} ; \mathbf{X})$ 
arbitrarily where there are taken a convex function $\phi(\mathbf{y} ; \mathbf{X})$ :

$\min _{\mathbf{X}_{(1)}, \ldots, \mathbf{X}_{(q)}} \phi\left(\mathbf{y} ; \mathbf{X}_{(1)}, \ldots, \mathbf{X}_{(q)}\right)=\phi\left(\mathbf{y} ; \hat{\mathbf{X}}_{(1)}, \ldots, \hat{\mathbf{X}}_{(q)}\right)$

In practice, in place of the arbitrary objective function the squared function is usually assumed (e.g. taking a normal distribution as a probabilistic model of measurement errors). Such special case of the parameters estimation method in the split functional model is called the squared $\mathrm{M}_{\text {split(q) }}$ estimation. The optimization criterion of squared $\mathrm{M}_{\text {split(q) }}$ estimation can be written in the following form (Wiśniewski, 2010):

$$
\begin{aligned}
\min _{\mathbf{x}_{(1)}, \ldots, \mathbf{x}_{(q)}} \phi\left(\mathbf{y} ; \mathbf{X}_{(1)}, \ldots, \mathbf{X}_{(q)}\right) & =\min _{\mathbf{x}_{(1)}, \ldots, \mathbf{X}_{(q)}} \sum_{i=1}^{n} p_{i}^{q} v_{i(1)}^{2} \ldots v_{i(q)}^{2}= \\
& =\phi\left(\mathbf{y} ; \hat{\mathbf{X}}_{(1)}, \ldots, \hat{\mathbf{X}}_{(q)}\right)
\end{aligned}
$$

where $\quad p_{i}=\frac{1}{m_{h_{i}}^{2}} \quad$ indicates the weight of observation $y_{i}$, and $m_{h_{i}}$ indicates the mean error of the height differences between two points. In the leveling network the square of mean error of height differences can be defined as $m_{h_{i}}^{2}=\frac{D_{i}}{D_{n o r m}} m_{h / k m}^{2}$, where $D_{i}$ - denote leveling strings length [km], $D_{\text {norm }}$ - is the normative length $(1 \mathrm{~km})$ of leveling strings and, $m_{h / \mathrm{km}}$ - indicates accuracy of the $1 \mathrm{~km}$ leveling network measurement $[\mathrm{mm}]$ (standard deviation). It is noteworthy that the least squares method, which minimize the objective function $\phi(\mathbf{y}, \mathbf{X})=\sum_{i=1}^{n} p_{i} v_{i}^{2}, \quad$ is a special case of the optimization criterion (11).

To solve the optimization problem (11) Newton method can be used (Teunissen, 1990; Wisniewski, 2009a). To determine the $M_{\text {split(q) }}$ estimators the gradient (12) and hessian (13) of the objective function $\quad \phi\left(\mathbf{y} ; \mathbf{X}_{(1)}, \ldots, \mathbf{X}_{(q)}\right)=\sum_{i=1}^{n} p_{i}^{q} v_{i(1)}^{2} \ldots v_{i(q)}^{2} \quad$ are calculated $(l=1, \ldots, q)$ :

$$
\begin{aligned}
\mathbf{g}_{(l)} & \left(\mathbf{X}_{(1)}, \ldots, \mathbf{X}_{(q)}\right)=\frac{\partial}{\partial \mathbf{X}_{(l)}} \phi\left(\mathbf{y}, \mathbf{X}_{(1)}, \ldots, \mathbf{X}_{(q)}\right)= \\
= & \frac{\partial \mathbf{v}_{(l)}}{\partial \mathbf{X}_{(l)}} \frac{\partial}{\partial \mathbf{v}_{(l)}} \phi\left(\mathbf{y}, \mathbf{X}_{(1)}, \ldots, \mathbf{X}_{(q)}\right)= \\
& =2 \mathbf{A}^{T} \mathbf{w}_{(l)}\left(\overline{\mathbf{v}}_{(k<l)}, \overline{\mathbf{v}}_{(k>l)}\right) \mathbf{P}_{\mathbf{y}} \mathbf{v}_{(l)}
\end{aligned}
$$

$$
\begin{aligned}
\mathbf{H}_{(l)}\left(\mathbf{X}_{(1)}, \ldots, \mathbf{X}_{(q)}\right) & =\frac{\partial^{2}}{\partial \mathbf{X}_{(l)} \partial \mathbf{X}_{(l)}^{T}} \phi\left(\mathbf{y} ; \mathbf{X}_{(1)}, \ldots, \mathbf{X}_{(q)}\right)= \\
& =2 \mathbf{A}^{T} \mathbf{w}_{(l)}\left(\overline{\mathbf{v}}_{(k<l)}, \overline{\mathbf{v}}_{(k>l)}\right) \mathbf{P}_{\mathbf{y}} \mathbf{A}
\end{aligned}
$$

where

$\mathbf{w}_{(l)}\left(\overline{\mathbf{v}}_{(k<l)}, \overline{\mathbf{v}}_{(k>l)}\right)=\operatorname{Diag}\left[\prod_{k=1, k \neq l}^{q} v_{1(k)}^{2}, \ldots, \prod_{k=1, k \neq l}^{q} v_{n(k)}^{2}\right]$ indicates the cross - weighting matrices (Wisniewski, 2010), and $\mathbf{P}_{\mathbf{y}}=\operatorname{Diag}\left[p_{1}^{q}, \ldots, p_{n}^{q}\right]$ indicates the weight matrices of observation. Then the iterative process of the squared $\mathrm{M}_{\text {split(q) }}$ estimation for $j=1, \ldots, m$, is as follows (Wiśniewski, 2010):

$\mathbf{X}_{(l)}^{j}=\mathbf{X}_{(l)}^{j-1}+d \mathbf{X}_{(l)}^{j}$
$\mathbf{v}_{(l)}^{j}=\mathbf{A} \mathbf{X}_{(l)}^{j}-\mathbf{y}$

where

$$
\begin{gathered}
d \mathbf{X}_{(1)}^{j}=-\left[\mathbf{H}_{(1)}\left(\mathbf{X}_{(1)}^{j-1}, \mathbf{X}_{(2)}^{j-1}, \ldots, \mathbf{X}_{(q)}^{j-1}\right)\right]^{-1} \mathbf{g}_{(1)}\left(\mathbf{X}_{(1)}^{j-1}, \mathbf{X}_{(2)}^{j-1}, \ldots, \mathbf{X}_{(q)}^{j-1}\right) \\
d \mathbf{X}_{(2)}^{j}=-\left[\mathbf{H}_{(2)}\left(\mathbf{X}_{(1)}^{j}, \mathbf{X}_{(2)}^{j-1}, \ldots, \mathbf{X}_{(q)}^{j-1}\right)\right]^{-1} \mathbf{g}_{(2)}\left(\mathbf{X}_{(1)}^{j}, \mathbf{X}_{(2)}^{j-1}, \ldots, \mathbf{X}_{(q)}^{j-1}\right) \\
\vdots \mathbf{X}_{(q)}^{j}=-\left[\mathbf{H}_{(q)}\left(\mathbf{X}_{(1)}^{j}, \mathbf{X}_{(2)}^{j}, \ldots, \mathbf{X}_{(q)}^{j-1}\right)\right]^{-1} \mathbf{g}_{(q)}\left(\mathbf{X}_{(1)}^{j}, \mathbf{X}_{(2)}^{j}, \ldots, \mathbf{X}_{(q)}^{j-1}\right)
\end{gathered}
$$

The solution is obtained iteratively, where the results of the least squares used as the first approximation estimators (startup items). A characteristic property is that the observation vector includes the measurements results from all measurement epochs, whereas the parameters in the split functional model are vectors

$$
\begin{aligned}
\mathbf{X}_{(q)}= & {\left[H_{K_{1}}, H_{K_{2}}, H_{A}, H_{B}, H_{C}, H_{D},\right.} \\
& \left.H_{E}, H_{S T_{1}}, H_{S T_{2}}, H_{H_{1}}, H_{S T_{3}}, H_{H_{2}}\right]^{T},
\end{aligned}
$$

containing the points heights in the $q$ measurements epochs. Another important feature of the $M_{\text {split }}$ estimation is that during the estimation, the observations are "intrinsically" assigned to the corresponding functional model on the basis of the cross - weighting matrix. This is important in the cases where a set of observations is a set of unrecognized implementation of several random variables. This method can also be applied in the case of the observations assignment to a particular random variable is known e.g., the geodetic network measurements in several measurement epochs. In our case study the number of measurement epochs is $q=5$.

\section{DESCRIPTION OF THE TESTED OBJECT}

The control network considered was designed in the area of the Old Town in Olsztyn near the Olsztyn Castle. In this study we consider the geodetic network as absolute. The control network consists of two reference points ( $R_{p}$ and $R_{k}$ ) and twelve controlled points $\left(K_{1}, K_{2}, A, B, C, D, E, S T_{1}, S T_{2}, S T_{3}\right.$, 


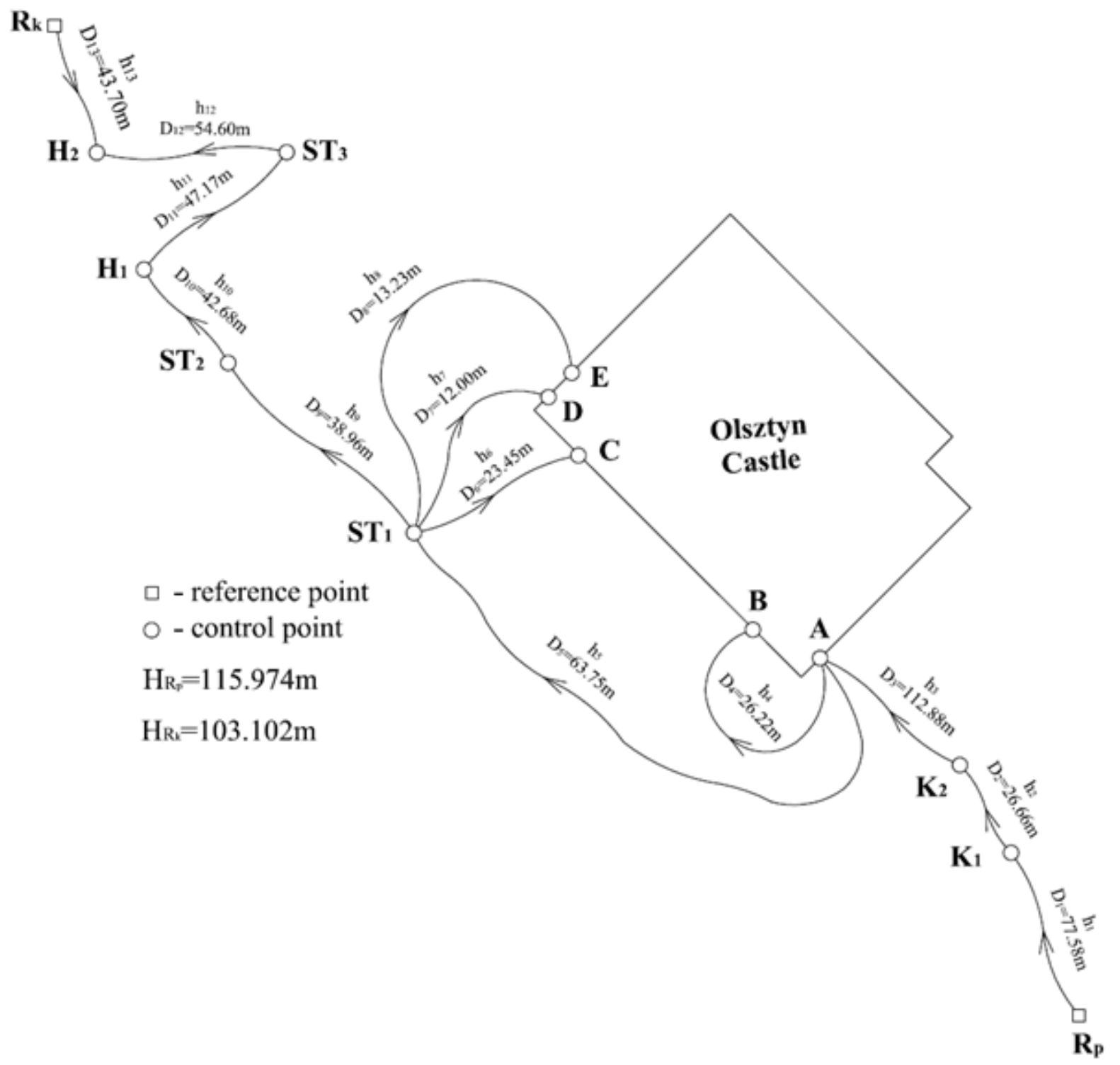

Fig. 2 The sketch of the control network to monitor the Olsztvn Castle.

$H_{1}$ and $H_{2}$ ). Location of these points in the area of the Olsztyn Old Town is depicted in Figure 2. Figure 2 presents also the geometry of the levelling control network. The sketch contains also the information about the number of the observations, the coordinates of the reference points and the leveling strings length. To perform the empirical analysis the results from five field campaign conducted between 2013 and 2015 were taken. Precise levelling instrument - Leica DNA 03 was used to collect the height differences between points. The precision of the one kilometer length leveling network obtained with this instrument is estimated at the level of 0.3 millimeter (standard deviation). The results of the field measurements of the all campaigns are presented in Table 1. All the values presented are in meters.

The results of the estimation parameters method in the split functional model of the geodetic observations were compared with the results obtained from the well-known least squares estimation which solving optimization criterion $\min _{\mathbf{x}} \sum_{i=1}^{n} p_{i} v_{i}^{2}$. The empirical analyzes are performed in three different scenarios:

Scenario I. The computations of $\mathrm{M}_{\text {split(q) }}$ estimates and LS estimates were performed using real observations of the monitored network.

Scenario II: It was assumed that the displacement of controlled point $C$ has been constant over all measurement epochs. Simulated values of the height point $C$ change relative to the first measurement period are respectively $\Delta_{1-2}^{C}=-0.009, \Delta_{1-3}^{C}=-0.013$, $\Delta_{-4}^{C}=-0.016$ and $\Delta_{1-5}^{C}=-0.019$. The modified values of the observations related to that point in the different measurement epochs are as follows i.e., $h_{6}^{\text {Epoch } 2}=1.14626, h_{6}^{\text {Epoch }}{ }^{3}=1.14208, h_{6}^{\text {Epoch }}{ }^{4}=1.13919$ and $h_{6}^{\text {Epoch }} 5=1.13666$. 
Table 1 The results of the precise leveling measurements obtained in the five campaigns.

\begin{tabular}{cccccc}
\hline & Epoch 1 & Epoch 2 & Epoch 3 & Epoch 4 & Epoch 5 \\
\hline & 21 November 2013 & 28 March 2014 & 1 July 2014 & 10 October 2014 & 9 January 2015 \\
\hline$h_{1}$ & 0.70511 & 0.70519 & 0.70537 & 0.70532 & 0.70511 \\
$h_{2}$ & -0.35989 & -0.35930 & -0.36015 & -0.36050 & -0.35987 \\
$h_{3}$ & -2.16602 & -2.16712 & -2.16636 & -2.16565 & -2.16602 \\
$h_{4}$ & -1.36668 & -1.36673 & -1.36692 & -1.36681 & -1.3667 \\
$h_{5}$ & -4.75371 & -4.75342 & -4.75365 & -4.75382 & -4.75382 \\
$h_{6}$ & 1.15542 & 1.15526 & 1.15508 & 1.15519 & 1.15566 \\
$h_{7}$ & -2.57547 & -2.57555 & -2.57592 & -2.57459 & -2.57532 \\
$h_{8}$ & -3.12253 & -3.12257 & -3.12220 & -3.12285 & -3.12238 \\
$h_{9}$ & -2.54677 & -2.54554 & -2.54646 & -2.54736 & -2.54714 \\
$h_{10}$ & -1.94240 & -1.94162 & -1.94123 & -1.94097 & -1.94203 \\
$h_{11}$ & -0.57261 & -0.57240 & -0.57222 & -0.57361 & -0.57246 \\
$h_{12}$ & -1.25550 & -1.25603 & -1.25629 & -1.25462 & -1.25562 \\
$h_{13}$ & -0.01861 & -0.01850 & -0.01878 & -0.01891 & -0.01849 \\
\hline
\end{tabular}

Table 2 The results from the least squares method and $\mathrm{M}_{\text {split (q) }}$ estimation.

\begin{tabular}{|c|c|c|c|c|c|c|c|c|c|c|}
\hline \multirow{2}{*}{$\begin{array}{l}\text { No. } \\
\text { points }\end{array}$} & \multicolumn{5}{|c|}{ Least Squares Estimation } & \multicolumn{5}{|c|}{$\mathrm{M}_{\text {split(q) }}$ estimation } \\
\hline & $\hat{\mathbf{X}}_{(1)}$ & $\hat{\mathbf{X}}_{(2)}$ & $\hat{\mathbf{X}}_{(3)}$ & $\hat{\mathbf{X}}_{(4)}$ & $\hat{\mathbf{X}}_{(5)}$ & $\hat{\mathbf{X}}_{(1)}$ & $\hat{\mathbf{X}}_{(2)}$ & $\hat{\mathbf{X}}_{(3)}$ & $\hat{\mathbf{X}}_{(4)}$ & $\hat{\mathbf{X}}_{(5)}$ \\
\hline$K_{1}$ & 116.6793 & 116.6792 & 116.6794 & 116.6794 & 116.6793 & 116.6792 & 116.6789 & 116.6793 & 116.6793 & 116.6800 \\
\hline$K_{2}$ & 116.3195 & 116.3198 & 116.3193 & 116.3189 & 116.3195 & 116.3192 & 116.3191 & 116.3193 & 116.3195 & 116.3202 \\
\hline$A$ & 114.1537 & 114.1527 & 114.1529 & 114.1533 & 114.1538 & 114.1535 & 114.1527 & 114.1533 & 114.1534 & 114.1531 \\
\hline$B$ & 112.7870 & 112.7859 & 112.7860 & 112.7865 & 112.7871 & 112.7868 & 112.7859 & 112.7866 & 112.7866 & 112.7862 \\
\hline$C$ & 110.5556 & 110.5545 & 110.5544 & 110.5547 & 110.5558 & 110.5550 & 110.5543 & 110.5552 & 110.5551 & 110.5553 \\
\hline$D$ & 106.8247 & 106.8237 & 106.8234 & 106.8249 & 106.8248 & 106.8241 & 106.8241 & 106.8244 & 106.8243 & 106.8250 \\
\hline$E$ & 106.2776 & 106.2766 & 106.2771 & 106.2767 & 106.2778 & 106.2776 & 106.2761 & 106.2777 & 106.2770 & 106.2769 \\
\hline$S T_{1}$ & 109.4001 & 109.3992 & 109.3993 & 109.3995 & 109.4002 & 109.3999 & 109.3988 & 109.4000 & 109.3997 & 109.3996 \\
\hline$S T_{2}$ & 106.8535 & 106.8536 & 106.8529 & 106.8522 & 106.8531 & 106.8527 & 106.8529 & 106.8530 & 106.8532 & 106.8541 \\
\hline$S T_{3}$ & 104.3387 & 104.3396 & 104.3395 & 104.3377 & 104.3389 & 104.3393 & 104.3383 & 104.3392 & 104.3387 & 104.3381 \\
\hline$H_{1}$ & 104.9112 & 104.9120 & 104.9117 & 104.9112 & 104.9112 & 104.9115 & 104.9108 & 104.9118 & 104.9113 & 104.9117 \\
\hline $\mathrm{H}_{2}$ & 103.0833 & 103.0835 & 103.0832 & 103.0831 & 103.0834 & 103.0832 & 103.0835 & 103.0831 & 103.0833 & 103.0835 \\
\hline
\end{tabular}

Scenario III: It is assumed that in addition to the point controlled $C$, the controlled point $B$ has been displaced by simulation as well. The simulated values of the height point $B$ change relative to the first measurement period are respectively $\Delta_{1-2}^{B}=-0.008$, $\Delta_{1-3}^{B}=-0.012, \quad \Delta_{-4}^{B}=-0.015$ and $\Delta_{1-5}^{B}=-0.018$. Thus, the actual values of the observations related to this point in the different measurement epochs are as follows i.e.. $h_{4}^{\text {Epoch } 2}=-1.37473, \quad h_{4}^{\text {Epoch } 3}=-1.37892$,

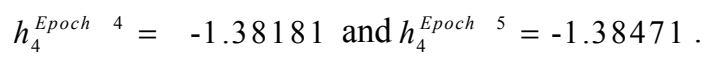

\section{RESULTS}

Table 2 contains the results from the least squares method and $\mathrm{M}_{\text {split(q) }}$ estimation. $\mathrm{M}_{\text {split(q) }}$ estimators were determined using all campaigns observation, whereas the LS estimates were calculated separately for each of the measurement epochs. The estimates obtained using $\mathrm{M}_{\text {split(q) }}$ in the Scenario I are similar to those obtained from least square method (Table 2). The results of estimating the shifts between parameters for specific measurement epochs, which are presented in Table 3, confirm the possibility of estimation of the reliable values of control point displacement by applying method of estimation of parameters in a split functional model. The individual values of the displacements of the controlled points were calculated in respect to the height estimators obtained for the first measurement epoch, ie.,

$\hat{\boldsymbol{\Delta}}_{(1)-(o)}=\hat{\mathbf{X}}_{(o)}-\hat{\mathbf{X}}_{(1)}$

where (o) indicates the number of the measurement period. 
Table 3 The displacements of the controlled points (Scenario I).

\begin{tabular}{|c|c|c|c|c|c|c|c|c|}
\hline \multirow[t]{2}{*}{$\begin{array}{l}\text { No. } \\
\text { points }\end{array}$} & \multicolumn{4}{|c|}{ Least Squares Estimation } & \multicolumn{4}{|c|}{$\mathrm{M}_{\text {split(q) }}$ estimation } \\
\hline & $\hat{\boldsymbol{\Delta}}_{1-2}$ & $\hat{\boldsymbol{\Delta}}_{1-3}$ & $\hat{\boldsymbol{\Delta}}_{1-4}$ & $\hat{\boldsymbol{\Delta}}_{1-5}$ & $\hat{\boldsymbol{\Delta}}_{1-2}$ & $\hat{\boldsymbol{\Delta}}_{1-3}$ & $\hat{\boldsymbol{\Delta}}_{1-4}$ & $\hat{\boldsymbol{\Delta}}_{1-5}$ \\
\hline$K_{1}$ & -0.0001 & 0.0001 & 0.0001 & 0.0000 & -0.0003 & 0.0001 & 0.0001 & 0.0008 \\
\hline$K_{2}$ & 0.0003 & -0.0002 & -0.0006 & 0.0000 & -0.0001 & 0.0001 & 0.0003 & 0.0010 \\
\hline$A$ & -0.0010 & -0.0008 & -0.0004 & 0.0001 & -0.0008 & -0.0002 & -0.0001 & -0.0004 \\
\hline$B$ & -0.0011 & -0.0010 & -0.0005 & 0.0001 & -0.0009 & -0.0002 & -0.0002 & -0.0006 \\
\hline$C$ & -0.0011 & -0.0012 & -0.0009 & 0.0002 & -0.0007 & 0.0002 & 0.0001 & 0.0003 \\
\hline$D$ & -0.0010 & -0.0013 & 0.0002 & 0.0001 & 0.0000 & 0.0003 & 0.0002 & 0.0009 \\
\hline$E$ & -0.0010 & -0.0005 & -0.0009 & 0.0002 & -0.0015 & 0.0001 & -0.0006 & -0.0007 \\
\hline$S T_{1}$ & -0.0009 & -0.0008 & -0.0006 & 0.0001 & -0.0011 & 0.0001 & -0.0002 & -0.0003 \\
\hline$S T_{2}$ & 0.0001 & -0.0006 & -0.0013 & -0.0004 & 0.0002 & 0.0003 & 0.0005 & 0.0014 \\
\hline$S T_{3}$ & 0.0009 & 0.0008 & -0.0010 & 0.0002 & -0.0010 & -0.0001 & -0.0006 & -0.0012 \\
\hline$H_{1}$ & 0.0008 & 0.0005 & 0.0000 & 0.0000 & -0.0007 & 0.0003 & -0.0002 & 0.0002 \\
\hline $\mathrm{H}_{2}$ & 0.0002 & -0.0001 & -0.0002 & 0.0001 & 0.0003 & -0.0001 & 0.0001 & 0.0003 \\
\hline
\end{tabular}

Table 4 The displacements of the controlled points (Scenario II).

\begin{tabular}{|c|c|c|c|c|c|c|c|c|}
\hline \multirow{2}{*}{$\begin{array}{l}\text { No. } \\
\text { points }\end{array}$} & \multicolumn{4}{|c|}{ Least Squares Estimation } & \multicolumn{4}{|c|}{$\mathrm{M}_{\text {split }}$ estimation } \\
\hline & $\hat{\boldsymbol{\Delta}}_{1-2}$ & $\hat{\boldsymbol{\Delta}}_{1-3}$ & $\hat{\boldsymbol{\Delta}}_{1-4}$ & $\hat{\boldsymbol{\Delta}}_{1-5}$ & $\hat{\boldsymbol{\Delta}}_{1-2}$ & $\hat{\Delta}_{1-3}$ & $\overline{\hat{\Delta}_{1-4}}$ & $\hat{\boldsymbol{\Delta}}_{1-5}$ \\
\hline$K_{1}$ & -0.0001 & 0.0001 & 0.0001 & 0.0000 & -0.0008 & -0.0008 & -0.0012 & -0.0009 \\
\hline$K_{2}$ & 0.0003 & -0.0002 & -0.0006 & 0.0000 & -0.0010 & -0.0008 & -0.0012 & -0.0011 \\
\hline$A$ & -0.0010 & -0.0008 & -0.0004 & 0.0001 & 0.0002 & 0.0003 & -0.0004 & 0.0004 \\
\hline$B$ & -0.0011 & -0.0010 & -0.0005 & 0.0001 & 0.0004 & 0.0004 & -0.0003 & 0.0006 \\
\hline$C$ & -0.0101 & -0.0142 & -0.0169 & -0.0188 & -0.0094 & -0.0139 & -0.0155 & -0.0184 \\
\hline$D$ & -0.0010 & -0.0013 & 0.0002 & 0.0001 & -0.0007 & -0.0008 & -0.0010 & -0.0010 \\
\hline E & -0.0010 & -0.0005 & -0.0009 & 0.0002 & 0.0007 & 0.0000 & -0.0009 & 0.0006 \\
\hline$S T_{1}$ & -0.0009 & -0.0008 & -0.0006 & 0.0001 & 0.0003 & 0.0000 & -0.0009 & 0.0002 \\
\hline$S T_{2}$ & 0.0001 & -0.0006 & -0.0013 & -0.0004 & -0.0011 & -0.0009 & -0.0012 & -0.0014 \\
\hline $\mathrm{ST}_{3}$ & 0.0009 & 0.0008 & -0.0010 & 0.0002 & 0.0010 & 0.0005 & 0.0001 & 0.0011 \\
\hline$H_{1}$ & 0.0008 & 0.0005 & 0.0000 & 0.0000 & 0.0000 & -0.0005 & -0.0010 & -0.0003 \\
\hline $\mathrm{H}_{2}$ & 0.0002 & -0.0001 & -0.0002 & 0.0001 & -0.0004 & -0.0002 & 0.0000 & -0.0003 \\
\hline
\end{tabular}

The results of the I Scenario clearly indicate that the tested object did not deform. To verify whether the proposed strategy will give the correct results, the geodetic network should deform, the authors introduced artificial displacements of the selected points. The observations of the I Scenario were modified in such a way that estimated height of the point C (the second Scenario) and B (option III) showed subsidence of selected points between measurement epochs. Obtained shifts of the controlled points for the II and III scenarios are presented in Tables 4 and 5. In these tables, the deformation indicators of the significantly displaced controlled points were bolded. The controlled points displacements in Scenarios II and III obtained by LS and $\mathrm{M}_{\text {split(q) }}$ methods, clearly show the deformation of the geodetic network. For both strategies, the obtained displacements of the controlled points are close to the theoretical-simulated values. Displacements of the artificially shifted points $C$ and $B$, are presented in Figure 3 and Figure 4 . The graphical interpretation of the obtained displacements clearly show the similarity of the results obtained by $\mathrm{M}_{\text {split(q) }}$ and least squares methods. The differences in the results of these methods are generally submillimetre. In the analyzed examples, the maximum difference between the calculated deformation indicators is $\hat{\boldsymbol{\Delta}}_{1-2}^{M \text { split }}-\hat{\boldsymbol{\Delta}}_{1-2}^{L S E}=1.8 \mathrm{~mm}$ for point $E$ in Scenario III.

The results show that $M_{\text {split(q) }}$ estimation may be considered as an alternative to the traditional methods applied to determine the controlled points displacements. This refers to the observations not disturbed by outliers. For those check new methods of geodetic observations adjustments were developed, focused on their robustness to outliers (see, eg., Baselga, 2011; Kamiński, 2011; Banaś and Ligas, 2014; Štroner et al., 2014; Třasák and Štroner, 2014; 
Table 5 The displacements of the controlled points (Scenario III).

\begin{tabular}{lrrrrrrrr}
\hline \multirow{2}{*}{$\begin{array}{c}\text { No. } \\
\text { points }\end{array}$} & \multicolumn{3}{c}{ Least Squares Estimation } \\
\cline { 2 - 9 } & $\hat{\boldsymbol{\Delta}}_{1-2}$ & \multicolumn{1}{c}{$\hat{\boldsymbol{\Delta}}_{1-3}$} & $\hat{\boldsymbol{\Delta}}_{1-4}$ & \multicolumn{1}{c}{$\hat{\boldsymbol{\Delta}}_{1-5}$} & $\hat{\boldsymbol{\Delta}}_{1-2}$ & \multicolumn{1}{c}{$\hat{\boldsymbol{\Delta}}_{1-3}$} & $\hat{\boldsymbol{\Delta}}_{1-4}$ & $\hat{\boldsymbol{\Delta}}_{1-5}$ \\
\hline$K_{1}$ & -0.0001 & 0.0001 & 0.0001 & 0.0000 & -0.0009 & -0.0009 & -0.0014 & -0.0011 \\
$K_{2}$ & 0.0003 & -0.0002 & -0.0006 & 0.0000 & -0.0011 & -0.0009 & -0.0014 & -0.0013 \\
$A$ & -0.0010 & -0.0008 & -0.0004 & 0.0001 & 0.0000 & 0.0000 & -0.0007 & 0.0001 \\
$B$ & $\mathbf{- 0 . 0 0 9 1}$ & $\mathbf{- 0 . 0 1 3 0}$ & $\mathbf{- 0 . 0 1 5 5}$ & $\mathbf{- 0 . 0 1 7 9}$ & $\mathbf{- 0 . 0 0 9 0}$ & $\mathbf{- 0 . 0 1 2 7}$ & $\mathbf{- 0 . 0 1 3 6}$ & $\mathbf{- 0 . 0 1 7 7}$ \\
$C$ & $\mathbf{- 0 . 0 1 0 1}$ & $\mathbf{- 0 . 0 1 4 2}$ & $\mathbf{- 0 . 0 1 6 9}$ & $\mathbf{- 0 . 0 1 8 8}$ & $\mathbf{- 0 . 0 0 9 3}$ & $\mathbf{- 0 . 0 1 3 8}$ & $\mathbf{- 0 . 0 1 5 5}$ & $\mathbf{- 0 . 0 1 8 4}$ \\
$D$ & -0.0010 & -0.0013 & 0.0002 & 0.0001 & -0.0006 & -0.0007 & -0.0010 & -0.0011 \\
$E$ & -0.0010 & -0.0005 & -0.0009 & 0.0002 & 0.0008 & 0.0001 & -0.0009 & 0.0006 \\
$S T_{1}$ & -0.0009 & -0.0008 & -0.0006 & 0.0001 & 0.0004 & 0.0000 & -0.0009 & 0.0002 \\
$S T_{2}$ & 0.0001 & -0.0006 & -0.0013 & -0.0004 & -0.0010 & -0.0009 & -0.0012 & -0.0015 \\
$S T_{3}$ & 0.0009 & 0.0008 & -0.0010 & 0.0002 & 0.0010 & 0.0005 & 0.0001 & 0.0011 \\
$H_{1}$ & 0.0008 & 0.0005 & 0.0000 & 0.0000 & 0.0002 & -0.0005 & -0.0010 & -0.0003 \\
$H_{2}$ & 0.0002 & -0.0001 & -0.0002 & 0.0001 & -0.0004 & -0.0001 & 0.0000 & -0.0003 \\
\hline
\end{tabular}

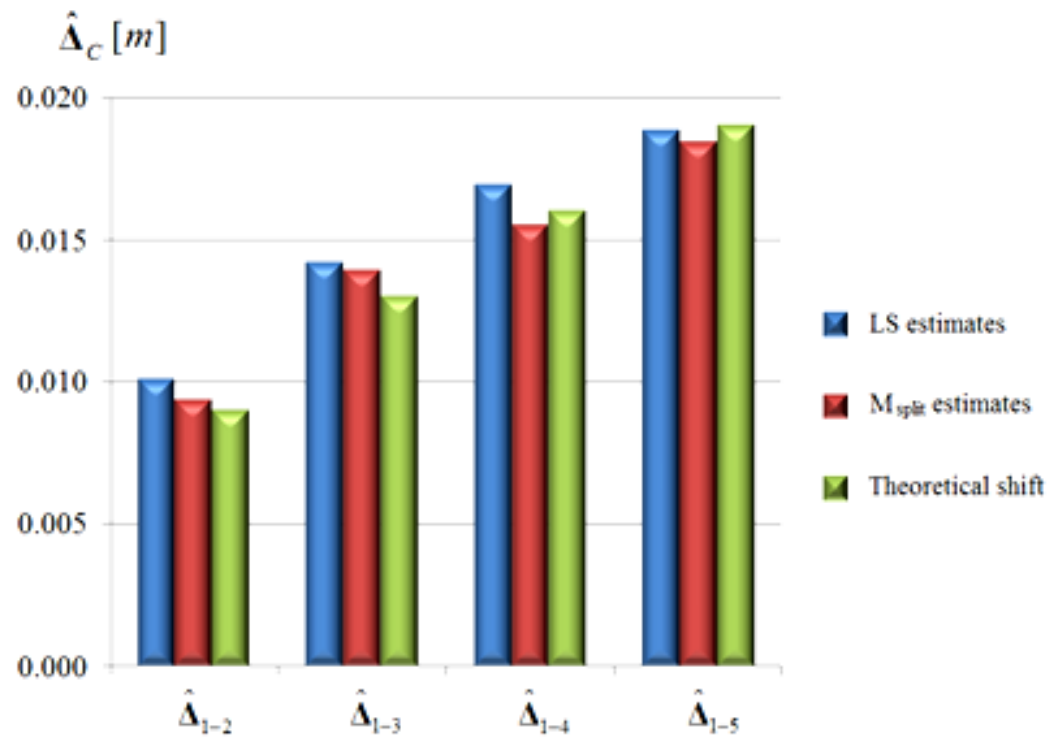

Fig. 3 The graphical presentation of the displacement of the C point obtained in II Scenario.

Wiśniewski, 2014; Durdag et al,. 2016; Osada et al., 2016). However it is noteworthy that in the papers (Zienkiewicz, 2014; Zienkiewicz and Baryła, 2015; Wisniewski and Zienkiewicz, 2016) shown that it is possible to obtain robust Msplit estimates by using a virtual functional model.

\section{CONCLUSION}

The experiments results presented in this paper show that the method based on the split of a conventional functional model can be an alternative to the traditional methods of estimation of the displacements. The results of the numerical tests show that in the case where the vector $y$ contains observations of several measurement epochs, the $M_{\text {split(q) }}$ estimation method gives similar results to the conventional least squares method. I Scenario show differences between the results of both methods on the submillimetre level. However, based on the results of the I Scenario, it is difficult to assess the efficacy of this method for displacements determination since, as the results showed, in our case there were not detected important shifts during five campaigns. As no large deformation has been observed during the campaign an artificial values for one (Scenario II) or two (Scenario III) controlled points have been introduced. In such scenario the results showed that displacements of the controlled points in a function of time and it is possible to detected by applying $\mathrm{M}_{\text {split(q) }}$ estimation method. The values of the height changes of the points $C$ and $B$ were similar to their theoretical-artificially introduced values. Thus, we can conclude that $\mathrm{M}_{\text {split(q) }}$ estimation method provides reliable results of controlled points displacements, a key factor for evaluation of the monitored object condition. 


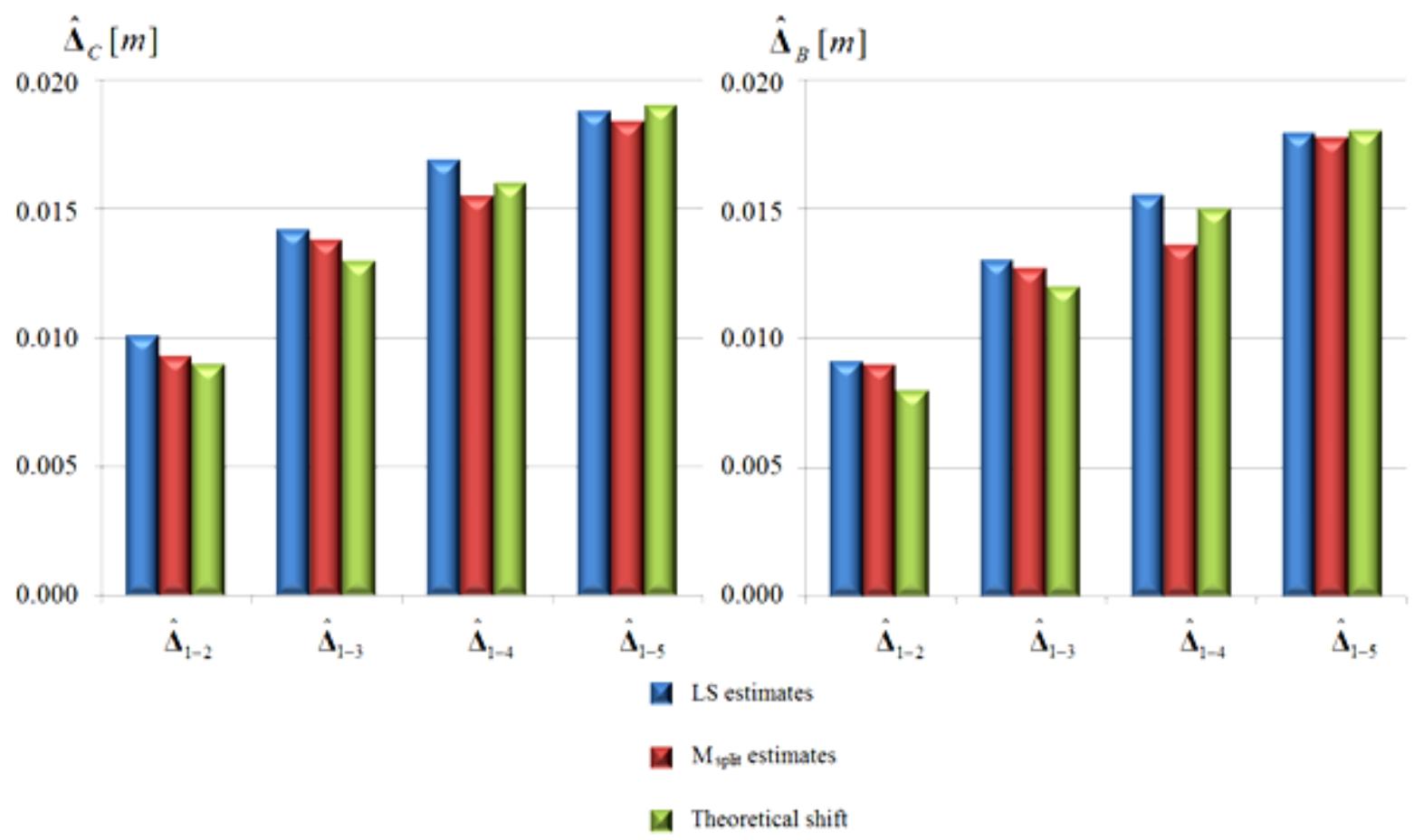

Fig. 4 The graphical presentation of the displacement of the C and B points obtained in III Scenario.

\section{REFERENCES}

Amiri-Simkooei, A., Alaei-Tabatabaei, S., Zangeneh-Nejad, F., and Voosoghi, B.: 2016, Stability analysis of deformation-monitoring network points using simultaneous observation adjustment of two epochs. Journal of Surveying Engineering, 143, No. 1. DOI: 10.1061/(ASCE)SU.1943-5428.0000195

Aydin, C.: 2012, Power of global test in deformation analysis. Journal of Surveying engineering, 138, No. $2,51-56$

DOI: $10.1061 /(A S C E) S U .1943-5428.0000064$

Banaś, M. and Ligas, M.: 2014, Empirical tests of performance of some M - estimators. Geodesy and Cartography, 63, No. 2, 127-146.

DOI: $10.2478 /$ geocart-2014-0010

Baselga, S.: 2011, Exhaustive search procedure for multiple outlier detection. Acta Geodaetica et Geophysica Hungarica, 46, No. 4, 401-416. DOI: 10.1556/AGeod.46.2011.4.3

Baselga, S., Garcia-Asenjo, L. and Garrigues, P.: 2015, Deformation monitoring and the maximum number of stable points method. Measurement, 70, 27-35. DOI: 10.1016/j.measurement.2015.03.034

Błaszczak-Bąk, W., Janowski, A., Kamiński, W. and Rapiński, J.: 2015, Application of the $\mathrm{M}_{\text {split }}$ method for filtering airborne laser scanning data-sets to estimate digital terrain models. Journal of Remote Sensing, 36, No. 9, 2421-2437.

DOI: $10.1080 / 01431161.2015 .1041617$

Caspary, W.F.: 1988, Concepts of network and deformation analysis. The University of New South Wales, Kensington.

Chen, Y.Q.: 1983, Analysis of deformation surveys - A generalized method. Ph.D. dissertation. Dept. of Geodesy and Geomatics Engineering, Technical Rep. No. 94, Univ. of New Brunswick, Fredericton, New Brunswick, Canada.
Cymerman, M., Duchnowski, R. and Kopiejczyk, A.: 2016, Selection of initial parameters in $\mathrm{R}$ - estimates applied to deformation analysis in leveling networks. Journal of Surveying Engineering, 142, No. 2. DOI: 10.1061/(ASCE)SU.1943-5428.0000151, 06015004

Czaplewski, K. and Wiśniewski, Z.: 2008, Hybrid M estimation in Maritime Navigation. Polish Journal of Environmental Studies, 17, No. 5A, 25-31.

Duchnowski, R.: 2010, Median-based estimates and their application in controlling reference mark stability. Journal of Surveying Engineering, 136, No. 2, 47-52. DOI: $10.1061 /(A S C E) S U .1943-5428.0000014$

Duchnowski, R.: 2011, Robustness of strategy for testing leveling mark stability based on rank tests. Survey Review, 43 No. 323, 687-699.

DOI: $10.1179 / 003962611 X 13117748892551$

Duchnowski, R. and Wiśniewski, Z.: 2012, Estimation of the Shift between parameters of functional models of geodetic observations by applying $\mathrm{M}_{\text {split }}$ estimation. Journal of Surveying Engineering, 138, No. 1, 1-8. DOI: 10.1061/(ASCE)SU.1943-5428.0000062

Duchnowski, R.: 2013, Hodges-Lehmann estimates in deformation analyses. Journal of Geodesy, 87 No. 10 12, 873-884. DOI: 10.1007/s00190-013-0651-2

Duchnowski, R. and Wiśniewski, Z.: 2014, Comparison of two unconventional methods of estimation applied to determine network point displacement. Survey Review, 46, No. 339, 401-405. DOI: $10.1179 / 1752270614$ Y.0000000127

Durdag, U.M., Hekimoglu, S. and Erdogan, B.: 2016, Outlier detection by using fault detection and isolation techniques in geodetic networks. Survey Review, 48, No. 351, 400-408. DOI: $10.1179 / 1752270615$ Y.0000000038

Erdogan, B. and Hekimoglu, S.: 2014, Effect of subnetwork configuration design on deformation analysis. Survey Review, 46, No. 335, 142-148. DOI: $10.1179 / 1752270613$ Y.0000000066 
Filipiak-Kowszyk, D. and Kamiński, W.: 2016, The use of free adjustment and $\mathrm{M}_{\text {split }}$ estimation for determination of the vertical displacements in unstable reference system. 2016 Baltic Geodetic Congress (BGS Geomatics), Gdansk.

DOI: 10.1109/BGC.Geomatics.2016.53

Ge, Y., Yuan, Y. and Jia, N.: 2013, More efficient methods among commonly used robust estimation methods for GPS coordinate transformation. Survey Review, 45, No. 330, 229-234. DOI: $10.1179 / 1752270612$ Y.0000000028

Hekimoglu, S., Erdogan, B. and Butterworth, S.: 2010, Increasing the efficacy of the conventional deformation analysis methods: alternative strategy. Journal of Surveying Engineering, 136, No. 2, 53-62. DOI: 10.1061/(ASCE)SU.1943-5428.0000018

Hekimoglu, S., Erenoglu, R.C., Sanli, D.U. and Erdogan, B.: 2011, Detecting configuration weaknesses in geodetic networks. Survey Review, 43 No. 323, 713-730. DOI: 10.1179/003962611X13117748892632

Hekimoglu, S. and Erdogan, B.: 2012, New median approach to define configuration weakness of deformation networks. Journal of Surveying Engineering, 138, No. 3, 101-108. DOI: 10.1061/(ASCE)SU.1943-5428.0000080

Janicka, J. and Rapiński, J.: 2013, $\mathrm{M}_{\text {split }}$ estimation of coordinates. Survey Review, 45, No. 331, 269-274. DOI: $10.1179 / 003962613 X 13726661625708$

Janowski, A. and Rapiński, J.: 2013, M - Split estimation in laser scanning data modeling. Journal of the Indian Society of Remote Sensing, 41, No. 1, 15-19. DOI: $10.1007 / \mathrm{s} 12524-012-0213-8$

Kamiński, W.: 2011, DiSTFAG method robust to gross errors in monitoring displacements and strains in unstable reference system. Geodesy and Cartography, 60 , No. 1, 21-33.

Kamiński, W. and Nowel, K.: 2013, Local variance factors in deformation analysis of non homogenous monitoring networks. Survey Review, 45, No. 328, 44-50. DOI: 10.1179/1752270612Y.0000000019

Nowel, K. and Kamiński, W.: 2013, Statistical significance of displacements in heterogeneous control networks. Geodesy and Cartography, 62, No. 2, 139-156.

Nowel, K. and Kamiński, W.: 2014, Robust estimation of deformation from observation differences for free control networks. Journal of Geodesy, 88, No.8, 749764. DOI: $10.1007 / \mathrm{s} 00190-014-0719-7$

Nowel, K.: 2015a, Investigating the efficacy of robust M estimation of deformation from observation differences. Survey Review, 48, No. 346, 21-30. DOI: $10.1080 / 00396265.2015 .1097585$

Nowel, K.: 2015b, Robust M - estimation in analysis of control network deformations: Classical and new method. Journal of Surveying Engineering, 141, No. 4. DOI: $10.1061 /(A S C E) S U .1943-5428.0000144$

Osada, E., Borkowski, A., Kurpiński, G., Oleksy, M. and Seta, M.: 2016, Fitting a precise levelling network to control points using a modified robust Huber's Mean Error Function. Journal of Surveying Engineering, 143, No. 1.

DOI: 10.1061/(ASCE)SU.1943-5428.0000201

Prószyński, W.: 1994, Criteria for internal reliability of linear least squares models. Bulletin Geodesique, 68, No. 3, 161-167. DOI: 10.1007/BF00808289

Prószyński, W.: 1997, Measuring the robustness potential of the least squares estimation: geodetic illustration. Journal of Geodesy, 71, No. 10, 652-659.

DOI: $10.1007 / \mathrm{s} 001900050132$
Štroner, M., Urban, R., Rys, P. and Balek, J.: 2014, Prague castle area local stability determination assessment by the robust transformation method. Acta Geodyn. Geomater., 11. No. 4 (176), 325-336.

DOI: $10.13168 /$ AGG.2014.0020

Sušić, Z., Batilović, M., Ninkov, T., Aleksić, I. and Bulatović, V.: 2015, Identification of movements using different geodetic methods of deformation analysis. GeodetskiVestnik, 59. No. 3, 537-553. DOI: 10.15292/geodetski-vestnik.2015.03.537-553

Teunissen, P.J.G.: 1990, Nonlinear least squares. Manuscripta Geodetica,15, No. 3, 137-150.

Třasák, P. and Štroner, M.: 2014, Outlier detection efficiency in the high precision geodetic network adjustment. Acta Geodaetica et Geophysica, 49, No. 2, 161-175. DOI: $10.1007 / \mathrm{s} 40328-014-0045-9$

Velsink, H.: 2015, On the deformation analysis of point fields. Journal of Geodesy, 25, No. 11, 1071-1087. DOI: $10.1007 / \mathrm{s} 00190-015-0835-\mathrm{Z}$

Wiśniewski, Z.: 2008, Split estimation of parameters in functional geodetic models. Technical Sciences, 11, 202-212.

Wiśniewsk,i Z.: 2009a, Estimation of parameters in a split functional model of geodetic observations $\left(\mathrm{M}_{\text {split }}\right.$ estimation). Journal of Geodesy, 83, No. 2, 105-120. DOI: 10.1007/s00190-008-0241-x

Wiśniewski, Z.: 2009b, $\mathrm{M}_{\text {split }}$ estimation. Part I: Theoretical foundation. Geodesy and Cartography, 58, No. 1, 321.

Wiśniewski, Z.: 2009c, $M_{\text {split }}$ estimation. Part II: Squared $M_{\text {split }}$ estimation. Numerical examples. Geodesy and Cartography, 58, No. 1, 23-48.

Wiśniewski, Z.: 2010, $\mathrm{M}_{\text {split(q) }}$ estimation: estimation of parameters in a multi split functional model of geodetic observations. Journal of Geodesy, 84, No. 6 , 355-372. DOI: 10.1007/s00190-010-0373-7

Wiśniewski, Z.: 2014, M-estimation with probabilistic models of geodetic observations. Journal of Geodesy, 88, No. $10,941-957$.

DOI: $10.1007 / \mathrm{s} 00190-014-0735-7$

Wiśniewski, Z. and Zienkiewicz, M.H.: 2016, Shift-M split $^{*}$ estimation in deformation analyses. Journal of Surveying Engineering, 142, No. 4. DOI: 10.1061/(ASCE)SU.1943-5428.0000183

Yetkin, M. and Berber, M.: 2013, Robustness analysis using the measure of external reliability for multiple outliers. Survey Review, 45, No. 330, 215-219. DOI: $10.1179 / 1752270612$ Y.0000000026

Zienkiewicz, M.H.: 2014, Application of $\mathrm{M}_{\text {split }}$ estimation to determine control points displacements in networks with unstable reference system. Survey Review, 47, No. 342, 174-180. DOI: $10.1179 / 1752270614$ Y.0000000105

Zienkiewicz, M.H. and Baryła, R.: 2015, Determination of vertical indicators of ground deformation in the Old and Main City of Gdansk area by applying unconventional method of robust estimation. Acta Geodyn. Geomater., 12, No. 3(179), 249-257. DOI: 10.13168/AGG.2015.0024

Zienkiewicz, M.H. and Bałuta, T.: 2013, Example of robust free adjustment of horizontal network covering detection of outlying points. Technical Sciences, 16, No. 3, 179-192. 